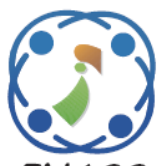

\title{
Image Edge Detection Based on Swarm Intelligence
}

\author{
Shahbaa I. Khaleel ${ }^{1 *}$ \\ ${ }^{1}$ Software Department, College of Computer Science and Mathematics, Mosul University, Iraq \\ * Corresponding author's Email: shahbaaibrkh@uomosul.edu.iq
}

\begin{abstract}
Since digital images have become an essential part in information systems and many other scientific applications, and due to the importance of some of these images in terms of the importance of the information they contain, there have been some problems in terms of the inability to determine the object in the image accurately or directly from the raw image. Because of the occurrence of errors affecting the process of transferring or taking these pictures, or there is a percentage of lack of clarity that leads to the loss of a part of the information, and this has led to the development and use of algorithms and methods that facilitate the process of detecting the edges of objects in the image and working to determine the spatial characteristics that are difficult to distinguish in sometimes. In this research, edge detection techniques and methods that depend on computational intelligence, and which are based in their work on biological content inspired by nature are presented. Two swarm intelligence methods represented by the beetle optimization method BSO and the butterfly optimization method BFM for conducting the edge detection process were presented in the images. In addition to developing a powerful edge detector based on the beetle and butterfly algorithms by improving the performance of the butterfly algorithm by choosing optimal values for its parameters that play an important role in finding the optimal solution. This was done by hybridizing it with the beetle algorithm that provides the butterfly algorithm with the best values for its parameters. The hybridization process was done in the first stage of the butterfly method, which is the initialization stage, this method called Beetle Butterfly Method BSO-BFM. All edge images that obtained using different swarm intelligence techniques of proposed system were compared by the reference edge image (ground truth) to ensure the performance, and accuracy of all methods used in the research, using mean square error MSE and root mean square error RMSE measurements as well as peak signal to noise ratio PSNR. From the results it turns out that the proposed hybrid method BSO-BFM is the best of all, this was evident through the values of the measures used here, as this method had the best values for the MSE measure, where its values ranged between $0.023375-0.466766$, and the RMSE measure, where the best values were obtained in this method, which is between $0.152889-0.216048$, and also for the PSNR measure, which ranges between $61.4398-64.443273$.
\end{abstract}

Keywords: Edge detection, Swarm intelligence, Beetle swarm optimization (BSO), Butterfly optimization.

\section{Introduction}

Edge detection processes are defined as the process of identifying spatial characteristics that are difficult to distinguish in some cases directly from raw images for the purpose of extracting formal compositions. The process of detecting edges is one of the basic steps in understanding and analyzing the image $[1,2]$. The vision based on distinguish objects begins with extracting local and general characteristics from the image of an object [3]. The edges are a characteristic inferred from object recognition systems, and the edge can be defined as changes or discontinuities in the intensity of the adjacent image cells in an image [4]. As these discontinuities "gaps" may occur due to differences in depth, changes in the nature of the surface of objects, differences in texture, changes in reflective properties, changes in lighting or color, or due to the formation of shadows that represent shapes and characteristics important to the image analysis [5]. Edge detection represents one of the basic methods in image processing and computer vision, these methods detect those discontinuities and changes in intensity of edges in images by looking on the 
relationship of each picture cell with its neighbors, and converting it into edges of image of the edge map, which is in the format binary, where the picture cells that are an edge with the value "one" are represented in white, and the picture cell that does not represent an edge with the value "zero" is represented in black [6]. The edge image resulting from edge detection algorithms is of great importance in computer vision systems, because the edges represent important information about the objects in the image, its type and location, and help explain the phenomena that form them. Therefore, these algorithms must be efficient and reliable, because their accuracy and effectiveness depend on them in the subsequent processing stages that depend on edges such as pattern recognition, image segmentation, and others [7].

Edge detection is a well-known and widely used technology in digital image processing. Where the edges represent important information about the nature of the images in the various fields used in them and through the difference in the characteristics of color intensity, color, texture or shadows, as well as the difference in the characteristics of the intensity of light during the image acquisition process, which is of great importance in aerial photographs, The low contrast shows little differences between the values of the image cells [8]. Therefore, good methods of detecting edges are a valuable tool that must be included in the methods of interpretation. There are a large number of image processing applications such as image matching, image segmentation, object marking, zoning, and others. Edge detection is used as a pre-processing for the purpose of extracting characteristics. Edges are used as an inference to "assess" movement and changes in successive images. Edge detection algorithms reduce image data to show edges only, and they describe the edges image in binary format, which reduces memory size and increases the speed of subsequent edgesdependent processing stages [9].

The intelligence of the SI, which is a branch of artificial intelligence, means the design of multi agent intelligent systems by drawing inspiration from the behavior collective of the insect community such as bees, ants, wasps, termites, and other societies of animal, flocks of fish and birds flocks $[10,11]$. Swarm intelligence describes the ability of groups of animals and insects to exhibit highly regular behaviors to solve complex problems that allow the group as a whole to accomplish tasks that exceed the ability of the single individual forming the group [12, 13]. This natural phenomenon is the inspiring factor for swarm intelligence systems, a class of algorithms that use and leverage naturally occurring swarm models and templates to solve computational problems $[14,15]$. Swarm intelligence and theory provide scope for detecting edges in images using different swarm techniques [16]. The most common method of improving an ant colony is to detect edges in various images. The ant colony algorithm focuses on a random building process that produces a solution probabilistically, through solving iteratively [17].

Here in this research, two intelligent algorithms were used in determining the edges in images, where two new methods of swarm intelligence were used in determining the edges with images, which are the beetle optimization method and the butterfly optimization method, and the two methods gave satisfactory results in determining the edges with images. In addition, the two methods were hybridized to improve performance by making an overlap between the two methods after knowing their behavior and strengths and overcoming their weaknesses. The beetle algorithm was used in the first stage of the butterfly method, which represents the initialization stage. By obtaining values for the variables of the butterfly method by adopting the beetle method, this overlap and hybridization gave distinct results in determining the edges with various images.

The research organization is as follows: The first section includes the introduction to the topic of the research, the second section includes previous works related to the topic, while the third section explains the method for optimizing the beetle, and the fourth section provides in detail the butterfly method, while the fifth section includes the proposed method for detecting the edges, which is hybridization between the beetle and butterfly methods, and the sixth section includes the proposed system. The experimental results are explained in detail in the seventh section, and in the final section the conclusions are written.

\section{Previous works}

Researchers Naveen Dagar and Pawan Dahiya presented a proposed methodology of edge detection based on Binary Particle Swarm Optimization that reduces the value of multiobjective fitness. This fitness value was formulated from five factors of cost as weighted sum, then all factors are associated by four edge validation techniques. The method that proposed was tested on a different set of images, and then compared with the classical methods in addition to the computational intelligent methods according to genetic algorithm GA and ant colony 
algorithm ACO. The proposed method outperformed all of these methods [9]. Researchers Alaa Eleyan and Muhammad Anwar presented a new method for detecting edges, and in the pre-processing stage, a discrete wavelet transfer (DWT) was used on the image whose edges were to be exposed, and to create sub-band images. And then apply Particle Swarm Optimization (PSO) to every one of the subband images. The processed image containing the detected edges is obtained by reconstructing the subband images using inverse transform. The work of the proposed method was evaluated on a set of images and the results were compared with the traditional methods by calculating the Peak Signal to Noise Ratio PSNR and Root Mean Square Error RMSE measures, and it became clear that the new method is better than other edge detection methods [7]. Where the RMSE values ranged between 0.39 0.77 , and the PSNR values for these methods ranged between 50.69 - 53.70. While in our research of the proposed methods (beetle BSO, butterfly BFM, Hybrid method BSO-BFM) for determining and detecting the edges of the images, the RMSE values ranged between $0.023375-0.101380$, and the PSNR values ranged between $58.071293-64.443273$, as it was found that our research methods were the best in their results.

A new objective function has been proposed by N. Dagar and P. Dahiya based on the optimization of an distinct particle swarm. This is to define the edges of the uncut image. This is done by creating a probability scale for the curve to be an edge. The proposed method was examined on eight images, and then compared this method with different techniques of edge detection, such as GA, canny, $\mathrm{ACO}$ and PSO, where the new algorithm gave a better result compared to other methods [18].

Researcher Dominik Weikert introduced an algorithm inspired by particle swarm optimization to define object contours in two dimensional environments. The particles search for the edge of the object within the image and then travel along its contour using the latest information they have about the locations inside and outside the object. With a robotic swarm, this could enable a robotic swarm to collectively define the boundaries of objects in the environment using internal sensors without the need for additional new imaging techniques. The experiments conducted have shown that the algorithm is working and promising results [19].

In this work, the butterfly algorithm was used, as well as the beetle algorithm, in addition to the fact that they were hybridized after studying their behavior, as these two algorithms are distinguished by their ability to reach the optimal solution, and overcome many of the obstacles found in the rest of the other swarm intelligence algorithms. As shown in the previous works mentioned, it was reached to define the edges well, but not ideally, since most of the methods used in the previous works, despite their strength, but during the work they fall in local solutions. Therefore, within our research, we decided to choose the best swarm intelligence algorithms that suit the application of edge identification because of their sensitivity with their parameters, where when choosing good values, optimal solutions were reached. As a result of merging and hybridizing between these two algorithms, the best results were reached, which were shown from the results of the measures used.

\section{The proposed system}

Edge detection processes are the process of identifying spatial features that are difficult to distinguish in some cases directly from raw images for the purpose of extracting formalism compositions. The process of detecting edges is one of the basic steps in understanding and analyzing the image. The edge detection process often includes the processes of identifying and isolating the edges of a digital image using digital image processing algorithms and methods.

Here in this research an efficient system was proposed to perform the edge detection process in various images, where two intelligent swarm algorithms were used to identify the edges in images, and this was done by using two new methods of swarm intelligence methods in determining the edges with images, which are the beetle optimization method and the butterfly optimization method and gave the two methods Satisfactory results in determining the edges in images, by calculating several performance measures of the resulting image, which are RMSE, PSNR, and MSE as shown in the Fig. 1.

In addition, the two methods were hybridized to improve performance by making an overlap between the two methods after knowing their behavior and their strengths. The beetle algorithm was used in the first stage of the butterfly algorithm, which represents the initialization stage. By obtaining values for the variables of the butterfly method by adopting the beetle method, this overlap and hybridization gave distinct results in identifying the edges with various images, which is clearly evident from the image results obtained using the proposed method and also from the values of the calculated measures to measure the performance of the method, that shown in tables. 


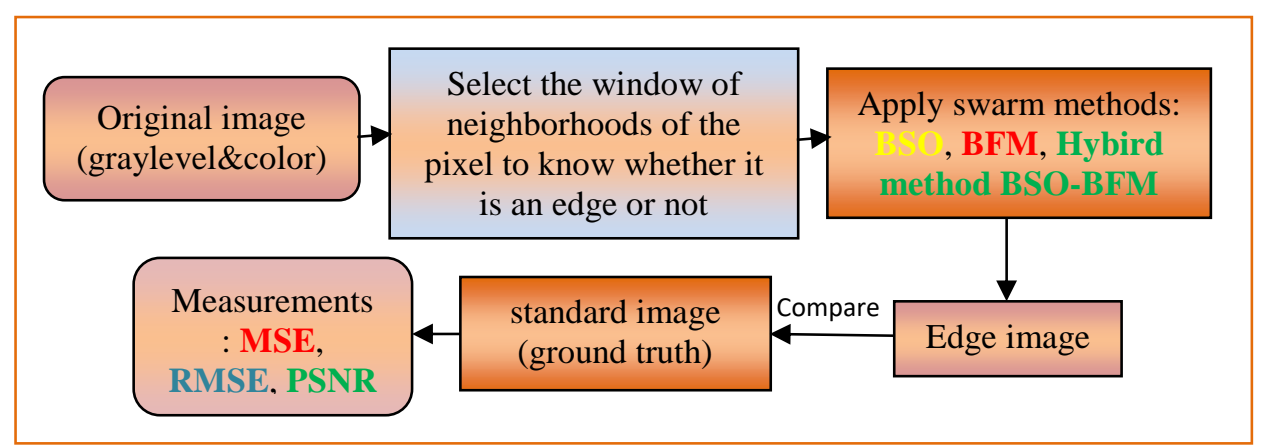

Figure. 1 Steps of proposed system

\section{Beetle optimization method}

The work of this algorithm is similar to the work of the particle algorithm where the beetles share information, but the direction and distance of the beetles are determined by the values of the velocity and the intensity of the information captured by the antennas of the beetles. Each beetle represents a possible solution to the problem, and each beetle has a fitness value determined by the fitness function [20]. This algorithm initially, initializes a set of random solutions, and then with every step or iteration the beetle updates its location depending on its search mechanism that the beetle has, with the best solution currently available. When these two parts are linked, it not only speeds up the implementation of population's steps, but also reduces the likelihood of members of the population falling into local sites [21, 22]. The speed of the beetle is updated or modified by the direction of movement of the beetle which approaches the individual extremity in addition to the direction of the movement of the Beetle Swarm Optimization BSO which approaches the global maximum value. Updating the beetle's position is not only determined by updating its speed, but also by the strength of the information detected by its antennas [23]. The work of the algorithm is illustrated by the following equations [21]:

$$
\begin{gathered}
\text { position }_{i s}^{i t+1}=\text { position }_{i s}^{i t}+\gamma \text { velocity } \\
(1-\gamma) \mu_{i s}^{i t} \\
(1- \\
\text { velocity }_{i s}^{i t+1}=\text { wvelocity }_{i s}^{i t}+ \\
\text { co }_{1} \text { rand }_{1}\left(\text { pos }_{i s}^{i t}-\text { position }_{i s}^{i t}\right)+ \\
\text { co }_{2} \text { rand }_{2}\left(\text { pos }_{g s}^{i t}-\text { position }_{g s}^{i t}\right) \\
\mu_{i s}^{i t+1}=\delta^{i t} \times \text { velocity }_{i s}^{i t} \times \\
\operatorname{sign}\left(f\left(\text { position }_{r s}^{i t}\right)-f\left(\text { position }_{l s}^{i t}\right)\right)
\end{gathered}
$$

$$
\operatorname{position}_{r s}^{i t+1}=\text { position }_{r s}^{i t}+\text { velocity }_{i s}^{i t} \times \frac{\vec{d}}{2}
$$

$$
\text { position }_{l s}^{i t+1}=\text { position }_{l s}^{i t}-\text { velocity }_{i s}^{i t} \times \frac{\vec{d}}{2}
$$

Where it represent iteration time, $\omega$ are inertia weight and $\gamma$ are loosening factor, rand 1 and rand $_{2}$ are random value between 0 and $1, \mathrm{CO}_{1}$ and $\mathrm{CO}_{2}$ are parameters that index to the impact degree of global and individual extremes on the beetle. $\delta$ represent length of beetle's searching step, $\vec{d}$ is the possible distance to be detected by the antennas of the beetle. position $_{l s}$ and position $_{r s}$ are the left and right positions of beetle's antennae that detected. And finally $\operatorname{pos}_{i s}$ are Individual extreme and $\operatorname{pos}_{g s}$ are global extreme, velocity is beetle's velocity[20]. To find and detect the edges within the image, contiguous pixels are used in calculating the value of the new light intensity of the center of the selected window[24].

Here in this research, the neighbors of the selected pixel was used to find the edges of the image data, as the new value of the gray level (center pixel) was calculated for eight contiguous points around center pixel in four directions, and this value fit became the value of the fitness of the beetle, which facilitates obtaining the optimal solution to detect the edges compared to traditional methods. As shown by the following equation:

$$
\begin{gathered}
\text { fit }=\left|\operatorname{Im}_{i-1, j}-\operatorname{Im}_{i, j}\right|+\left|\operatorname{Im}_{i+1, j}-\operatorname{Im}_{i, j}\right|+ \\
\left|\operatorname{Im}_{i, j-1}-\operatorname{Im}_{i, j}\right|+\left|\operatorname{Im}_{i, j+1}-\operatorname{Im}_{i, j}\right|+ \\
\left|\operatorname{Im}_{i-1, j-1}-\operatorname{Im}_{i, j}\right|+\left|\operatorname{Im}_{i-1, j+1}-\operatorname{Im}_{i, j}\right|+ \\
\left|\operatorname{Im}_{i+1, j-1}-\operatorname{Im}_{i, j}\right|+\left|\operatorname{Im} m_{i+1, j+1}-\operatorname{Im}_{i, j}\right|
\end{gathered}
$$

Where $I m_{i, j}$ represent the center pixel at position $i, j$ within gray level image Im.

After initialize the position and velocity of beetles randomly, then calculate the value of fitness of each beetle that depend on both left and right position of it, by using current position to $p^{\circ} s_{i s}$ are Individual extreme and $\operatorname{pos}_{g s}$ are global extreme. The new value of the pixel that selected, is the 
optimal solution that obtained, to detect this is edge or not.

\section{Butterfly optimization method}

The Butterfly Optimization algorithm relies on models of butterfly biological behavior to find food sources and mate [25]. In nature, butterflies use their senses to find food, the most important senses are the smell that helps the butterfly to accomplish this work, as the butterfly generates the fragrance and its percentage is according to its fitness and this depends on the butterfly's movement from one location to another [26]. This method has three main stages since all butterflies are supposed to emit some fragrance, causing the butterflies to be attracted to each other. Each butterfly will move randomly or move towards the best butterfly (which emits more scent) and finally, the landscape greatly influences the search field on the level of stimulation intensity of each butterfly [26]. These principles are embodied in three main stages for this algorithm: the initialization stage, the iteration stage, and the final stage. Where all the algorithm parameters are initialized, then the search phase is carried out in an iterative manner, and finally the optimal solution is obtained [27].

In the preparation stage, the algorithm is equipped with the important parameters of determining the objective function that represents the perceived size of the fragrance, i.e. the extent of the strength of the butterflies' perception of the fragrance depending on the value of the stimulus intensity of the fragrance and in turn depends on the sensitivity modality (sensory) in addition to the power exponent based on the nature of the sensory modality which is determined or calculated the variation in the degree of absorption. The objective function is represented by the following equation [25]:

$$
\text { frag }=m S I^{p}
$$

Where $m$ : represent sensory modality the value between (0-1), SI : stimulus intensity and $p$ power exponent between $(0-\infty)$.

An initial group of butterflies is created, i.e. a primary community whose number does not change over the length of the implementation of the algorithm, and random locations for the butterflies are chosen at the beginning of the work, and after determining all these parameters, the initialization phase ends and moves to the second phase of the algorithm's work, which is the iteration phase where the algorithm is executed. With a number of iterations, the butterflies move in the solution area, meaning they change their locations. Then find the best solution by following global search and local search, and the decision is made based on the probability value of the butterfly's behavior towards local search or global search. And as shown by the following equations. Finally, when the best solutions are obtained, the algorithm ends [28].

for global search

$$
\begin{array}{r}
\operatorname{Pos}_{i}^{i t+1}=\operatorname{Pos}_{i}^{i t}+\left(r n^{2} \times \text { bestsol }^{*}-\operatorname{Pos}_{i}^{i t}\right) \times \\
\text { frag } \\
\text { fri }(8)
\end{array}
$$

for local search

$$
\begin{array}{r}
\operatorname{Pos}_{i}^{i t+1}=\operatorname{Pos}_{i}^{i t}+\left(r^{2} \times \operatorname{Pos}_{j}^{i t}-\operatorname{Pos}_{k}^{i t}\right) \times \\
\operatorname{frag}_{i}(9)
\end{array}
$$

Where $i t+1$ and it represent the current and update state of position, bestsol ${ }^{*}$ is the best solution in the space search, while $P_{o s}$ and $P_{o s}$ are two butterflies from population which selected randomly. Finally rn are random number from (0-1) [28]. The process of determining the edges of the images depends mainly on the principle of adjacent to the pixels, where a window consisting of a group of pixels is determined and the edge value of which depends on the values of the image intensity $[17,24]$. In this research, the contiguousness of the selected light point (pixel) was used to calculate or find the edges of the image data, as the new value of the gray scale (center pixel) was calculated for eight contiguous points, and this value became the value of the stimulus intensity of the fragrance of the butterfly, which facilitates obtaining the optimal solution to detect the edges compared to traditional methods. As shown by the following equation:

$$
\begin{gathered}
S I=\left|I g_{i-1, j}-I g_{i, j}\right|+\left|I g_{i+1, j}-I g_{i, j}\right|+ \\
\left|I g_{i, j-1}-I g_{i, j}\right|+\left|I g_{i, j+1}-I g_{i, j}\right|+ \\
\left|I g_{i-1, j-1}-I g_{i, j}\right|+\left|I g_{i-1, j+1}-I g_{i, j}\right|+ \\
\left|I g_{i+1, j-1}-I g_{i, j}\right|+\left|I g_{i+1, j+1}-I g_{i, j}\right|
\end{gathered}
$$

Where $I g_{i, j}$ represent the center pixel at position $i, j$ within gray level image Ig. Here in this research when implementing this algorithm on gray level and color images, the results were excellent and better than the beetle method.

The process of determining the edges of the images depends mainly on the principle of adjacent to the pixels, where a window consisting of a group of pixels is determined and the edge is found whose value depends on the values of the image intensity. When implementing this algorithm butterfly 
optimization method BFM on gray level and color images, the results were excellent and better than the beetle algorithm BSO.

\section{Beetle Butterfly Method BSO-BFM proposed to edges detection}

The beetle algorithm is similar in its work to the particle swarm algorithm, as it works on the same principle and that when calculating the velocity of the beetle's movement from one location to another, there are variables (parameters) within its equation $\mathrm{Co}_{1}, \mathrm{CO}_{2}$. These variables have a role in the velocity balance that directly affects the new location that the beetle will take, when It has the effect of determining the direction of movement of the beetle which approaches the individual extremes as well as the direction of the BSO movement which approaches the global maximum value. So these variables play an important role in determining the new sites that the beetle will take to reach the optimal solution.

The butterfly algorithm depends in its work on the principle of the ant algorithm, which in turn relies on the pheromone substance secreted by the ant to determine the correct path to reach the best solution. With this principle, the butterfly algorithm also works to reach the optimal solution by adopting the fragrance, and that the variables present with the intensity of stimulation of the butterfly have a role in determining the optimal path to reach the best solutions.

Therefore, the focus here in this research is to find the best values for the sensitivity modality (sensory) $m$ in addition to the power of the exponent $p$ on the basis of the nature of the sensory modality that determines or calculates the variance in the degree of absorption, as these two values are multiplied by the intensity of the stimulus, thus resulting in the strength of the butterflies' perception of the fragrance, which in turn enters In the calculation of the local and global locations of the butterfly. Which leads to speeding up the process of reaching the best solution.

Where the beetle algorithm was used in the first stage of the butterfly method represented by the initialization stage to find the best values for the parameters of the butterfly algorithm that shown in the following equation:

$$
\begin{aligned}
& \text { velocity }_{i s}^{i t+1}=\text { velocity }_{i s}^{i t}+m B \operatorname{rand}_{1}\left(\text { pos }_{i s}^{i t}-\right. \\
& \text { position } \left._{i s}^{i t}\right)+p B \operatorname{rand}_{2}\left(\text { pos }_{g s}^{i t}-\text { position }_{g s}^{i t}\right)(11)
\end{aligned}
$$

Because when setting the parameters of the butterfly algorithm, the best solutions are reached with this algorithm and not moving away from the solution by using the following objective function:

$$
f r a g=m B S I^{p B}
$$

Where here in this research the best values for the sensitivity modality as well as the power of the exponent were found, which play a very important role in the balance of the butterfly algorithm and give the best results. This was shown when applied BSO-BFM to the same images used in the research by using the beetle method and the butterfly method, where the best results were obtained using the hybrid method that gave the best values for the quality measures of the method represented by MSE RMSE PSNR as shown in the tables. Fig. 2 show flowchart of proposed method.

\section{Experimental results}

In this research, three intelligent swarm techniques are used that are efficient in conducting the edge detection process in various images, depending on the neighbors to decide whether the pixels are an edge or not. Where, at first, the beetle optimization method was used in the process of determining the edges of the image, which gave satisfactory results, as the principle of neighbors was used in determining the objective function of the algorithm in order to obtain the optimal solution in the fastest time and the fewest iterations. Then the butterfly optimization method was used for the same purpose, which is edge detection, and this method was better than the first method for edge detection. From the study of the behavior of these two methods, a hybrid method was proposed from the two above mentioned methods, where the first stage of the butterfly method was hybridized by using the beetle optimization method to obtain the important parameters in decision-making in the butterfly algorithm, which led to the improvement of the performance of the proposed method, as its results were better than The previous two methods.

The research methods were applied to the images used by many researchers to demonstrate and prove the efficiency of the methods used here. Where in the beginning the first method applied, the beetle optimization method in conducting the edge detection process on many images, including the first image (Lena image), and the results were satisfactory using the beetle method BSO where the measurements values of the performance of the method represented by the MSE measure were 0.046873 and the RMSE value is 0.216501 . The PSNR value was equal to 61.421608 . As for the 


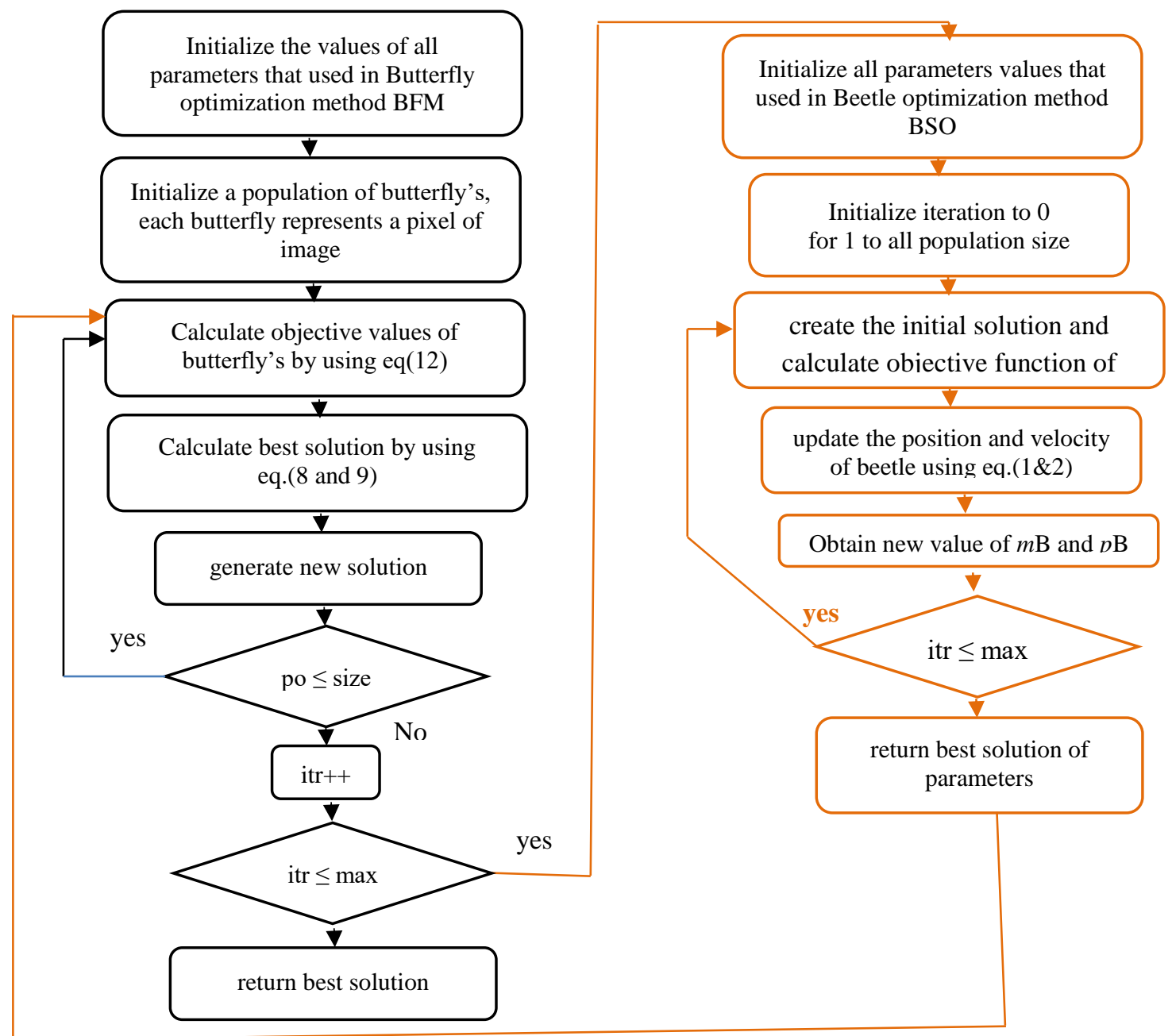

Figure. 2 The flowchart of the proposed method BSO-BFM

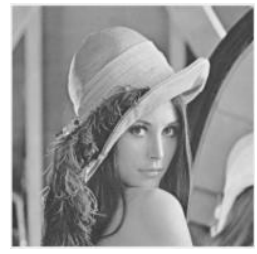

(a)

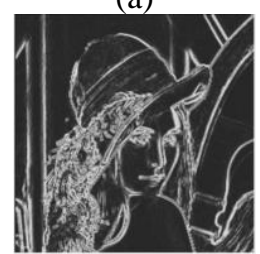

(d)

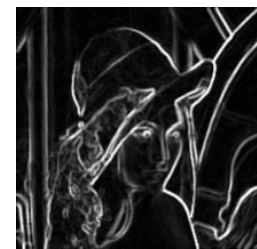

(b)

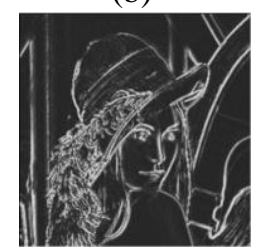

(e)

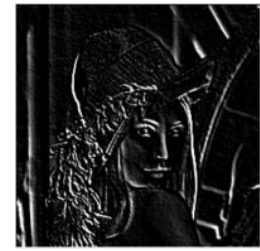

(c)

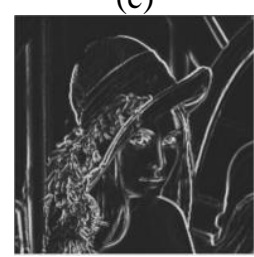

(f)
Figure. 3 Results of all methods for Lena image: (a) original image, (b) standard image (ground truth), (c) Sobel method, (d) BSO method, (e) BFM method, and (f) Hybird method BSO-BFM

butterfly method BFM, the performance improved and the measurements values were better, as the MSE value decreased and became 0.037450 , as well as the RMSE value became better 0.193521, and finally the PSNR measure was 62.396241, and when applying the proposed algorithm BSO-BFM the results were the best of all, where the values of the measurements are as follows: MSE: 0.029442 and the RMSE value: 0.171586 , the PSNR value: 63.441142, which is the best of all. Also, the research methods represented by the swarm intelligence algorithms were compared to the traditional methods, where the edges of this image were detected using sobel, and the values of the measurements were in this method as follows: MSE: 0.220466 RMSE: 0.469538 PSNR: 54.697392. And it became clear from these results that the research methods have proven their efficiency at work.

Fig. 3 shows the results of all methods for the grayscale image, Lena Image, and Table 1 shows the results of performance measures for the methods used in the research.

Fig. 4 shows the relationship between the performance measures used in the research to show the quality of the proposed method, where we notice that the values of the two scales MSE and RMSE start decreasing starting from the sobel method and 
Table 1. Performance Measures for Lena image

\begin{tabular}{|c|c|c|c|}
\hline Methods & MSE & $\boldsymbol{R M S E}$ & $\boldsymbol{P S N R}$ \\
\hline Sobel & 0.220466 & 0.469538 & 54.697392 \\
\hline BSO & 0.046873 & 0.216501 & 61.421608 \\
\hline BFM & 0.037450 & 0.193521 & 62.396241 \\
\hline BSO-BFM & 0.029442 & 0.171586 & 63.441142 \\
\hline
\end{tabular}

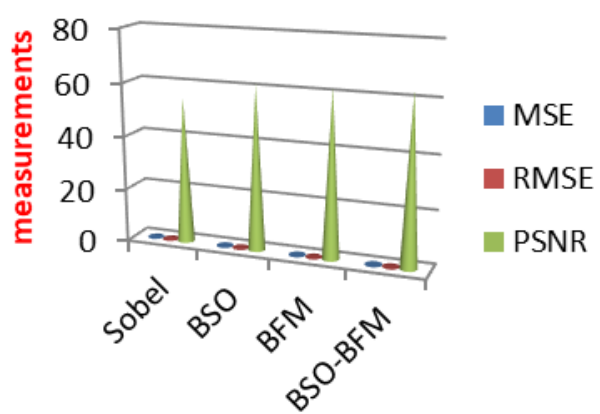

Figure. 4 MSE, RMSE, PSNR measures of all methods

ending with the proposed method BSO-BFM and this leads to an increase in the PSNR values because it is directly proportional to the previous two measures, and this indicates The strength of the method is to detect the edges of the image perfectly, and therefore the proposed method was the best among the methods.

When comparing this research work with the work of other researchers, we notice that our research methods are much better than other methods. This is evidenced by a comparison of this research methods with reference research methods in [7] Where the swarm intelligence algorithms were used, which is the particle swarm intelligence, which gave good results, from measures values. Where the RMSE values ranged between 0.39 - 0.77 , and the PSNR values for these methods ranged between 50.69 - 53.70. While in our research of the proposed methods (beetle BSO, butterfly BFM, Hybrid method BSO-BFM) for determining and detecting the edges of the images, the RMSE values ranged between $0.023375-0.101380$, and the PSNR values ranged between $58.071293-64.443273$, as it was found that our research methods were the best in their results. This is because efficient and sure swarm intelligence methods were used to reach the ideal solution. It is also when hybridization between the two methods of swarm intelligence we obtained the optimal results, the butterflies algorithm and the beetle algorithm to produce a new proposed method BSO-BFM that highly efficient.

Fig. 5 and 7 illustrate the results of the three research methods represented by the beetle method, the butterfly method, and the proposed hybrid method BSO-BFM for the baboon and the Jet images. In addition to Table 2 and 3, they show the

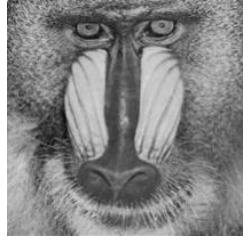

(a)

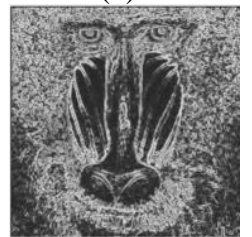

(d)

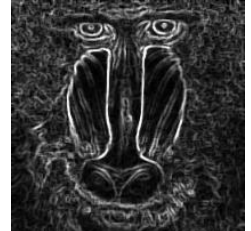

(b)

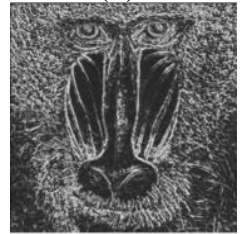

(e)

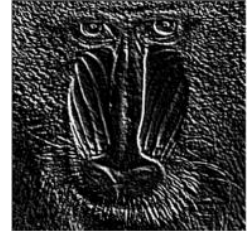

(c)

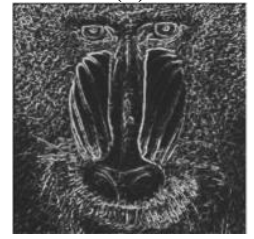

(f)
Figure. 5 Results of all methods for Baboon image:(a) original image, (b) ground truth, (c) Sobel method, (d) BSO method, (e)BFM method, and (f) Hybird method BSO-BFM

Table 2. Performance measures for Baboon image

\begin{tabular}{|c|c|c|c|}
\hline Methods & MSE & RMSE & PSNR \\
\hline Sobel & 0.375440 & 0.612732 & 52.385397 \\
\hline BSO & 0.101380 & 0.318402 & 58.071293 \\
\hline BFM & 0.065059 & 0.255066 & 59.997748 \\
\hline BSO-BFM & 0.037012 & 0.192386 & 62.447335 \\
\hline
\end{tabular}

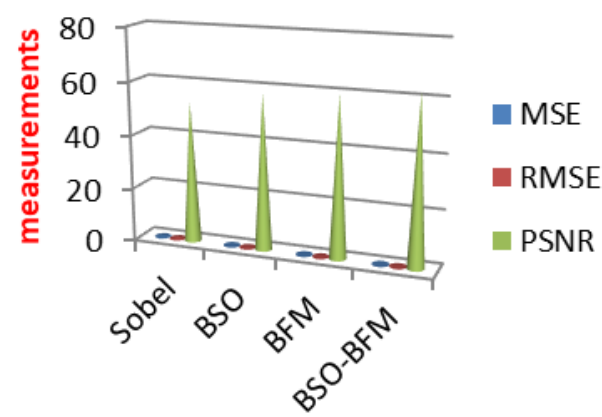

Figure. 6 MSE, RMSE, PSNR measures of all methods

values of the used methods performance measures. As it is clear from the results that the proposed hybrid method was the best among the research methods, and the best results were obtained after applying it to the images to identify and reveal the edges in them.

And also Fig. 6 shows the performance measures, MSE, RMSE, PSNR that used in research of all methods. Which proves that the proposed method was the best among the methods used to obtain the best values for all scales when applied to the standard picture baboon.

Fig. 8 shows the values of MSE, RMSE of all methods that applied on standard image Jet. It is clear that the proposed method gave the best results Also, research methods have been applied to color images to find or define the edges of these images. Fig. 9, 11-13 illustrate the results of the three research methods represented by the beetle method, 


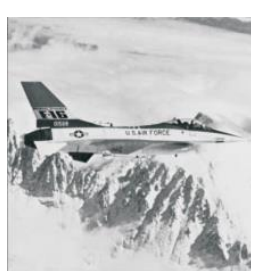

(a)

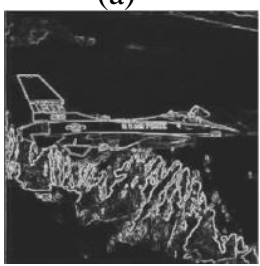

(d)

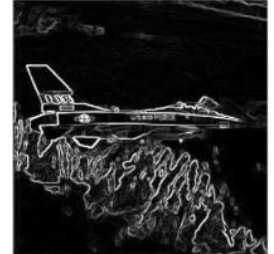

(b)

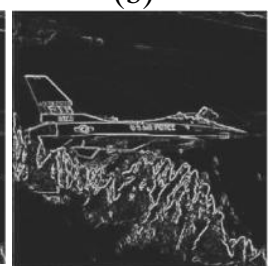

(e)

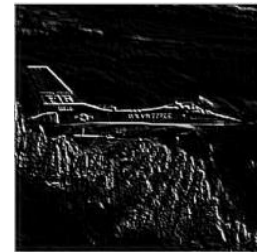

(c)

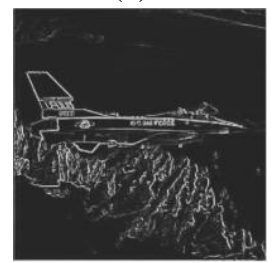

(f)
Figure. 7 Results of all methods for Jet image:(a) original image, (b) ground truth, (c) Sobel method, (d) BSO method, (e)BFM method, and (f) Hybird method BSO-

BFM

Table 3. Performance measures for Jet image

\begin{tabular}{|c|c|c|c|}
\hline Methods & MSE & $\boldsymbol{R M S E}$ & $\boldsymbol{P S N R}$ \\
\hline Sobel & 0.170389 & 0.412782 & 55.816393 \\
\hline BSO & 0.029626 & 0.172121 & 63.414130 \\
\hline BFM & 0.025674 & 0.160231 & 64.035850 \\
\hline BSO-BFM & 0.023375 & 0.152889 & 64.443273 \\
\hline
\end{tabular}

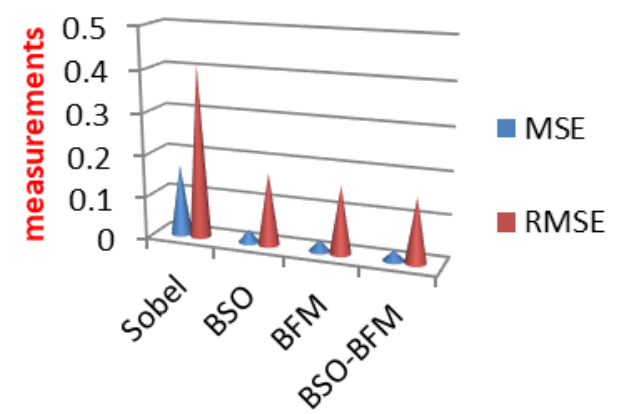

Figure. 8 MSE, RMSE measures of all methods

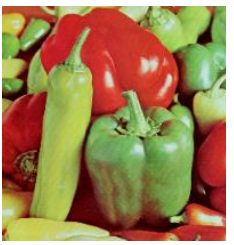

(a)

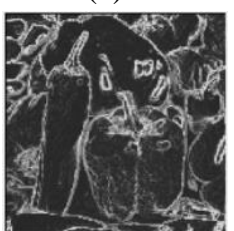

(d)

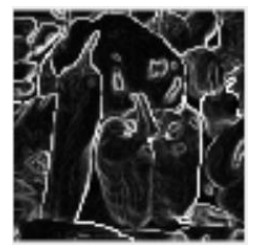

(b)

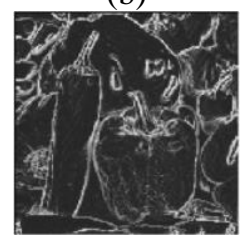

(e)

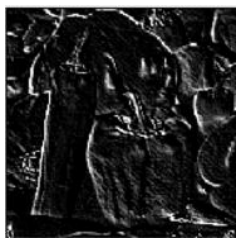

(c)

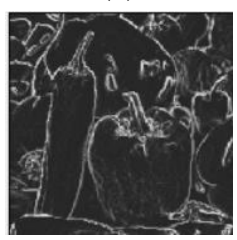

(f)
Figure. 9 Results of all methods for peppers image: (a) original image, (b) ground truth, (c) Sobel method, (d) BSO method, (e)BFM method, and (f) Hybird method BSO-BFM measures of the methods used are shown in Tables 4-7. Where it is clear from the results that the proposed hybrid method was the best among the research methods, and the best results were obtained after applying it to the color images to identify the edges in them. Fig. 10 shows the PSNR value of all method when applied on peppers image.

Fig. 14 shows the chart that explain the values of MSE, RMSE of all method that applied on flower2 image that proved the proposed method is the best.

Table 4. Performance measures for peppers image

\begin{tabular}{|c|l|c|c|}
\hline Methods & \multicolumn{1}{|c|}{ MSE } & RMSE & PSNR \\
\hline Sobel & 0.311033 & 0.557703 & 53.2027 \\
\hline BSO & 0.036186 & 0.190228 & 62.5453 \\
\hline BFM & 0.03004 & 0.173324 & 63.3536 \\
\hline BSO-BFM & 0.027424 & 0.165603 & 63.7494 \\
\hline
\end{tabular}

PSNR

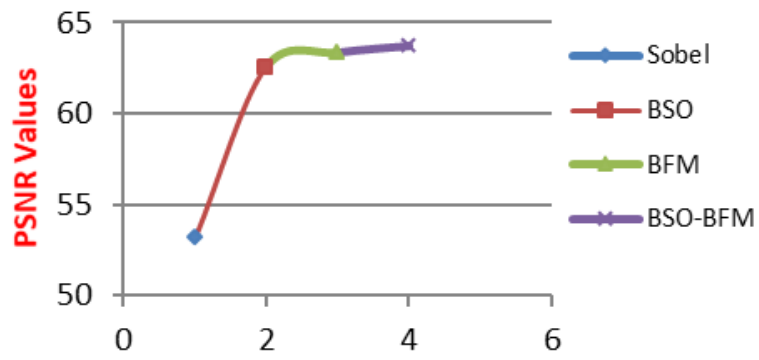

Figure. 10 Value of PSNR of all methods

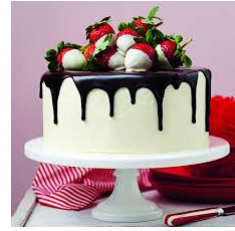

(a)

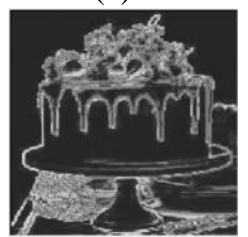

(d)

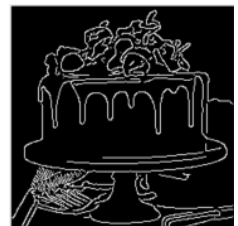

(b)

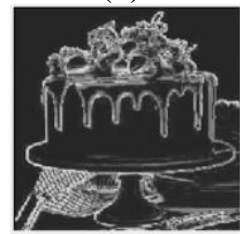

(e)

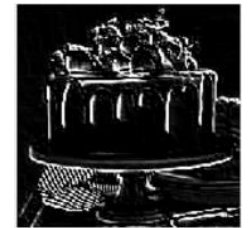

(c)

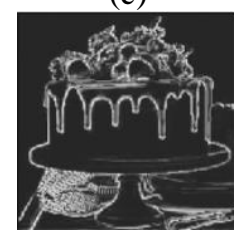

(f)
Figure. 11 Results of all methods for cake image:(a) original image, (b) ground truth, (c) Sobel method, (d) BSO method, (e)BFM method, and (f) Hybird method BSO-BFM

Table 5. Performance measures for cake image

\begin{tabular}{|c|l|l|l|}
\hline Methods & \multicolumn{1}{|c|}{ MSE } & \multicolumn{1}{|c|}{ MSE } & \multicolumn{1}{c|}{ PSNR } \\
\hline Sobel & 0.135328 & 0.36787 & 56.8169 \\
\hline BSO & 0.078379 & 0.279962 & 59.1888 \\
\hline BFM & 0.04318 & 0.207798 & 61.778 \\
\hline BSO-BFM & 0.027442 & 0.165656 & 63.746678 \\
\hline
\end{tabular}

the butterfly method, and the proposed hybrid method for color images. The values of the 


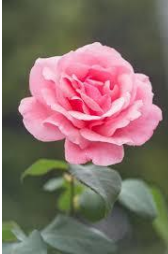

(a)

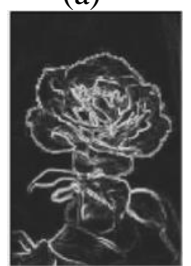

(d)

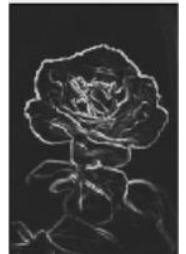

(b)

(e)

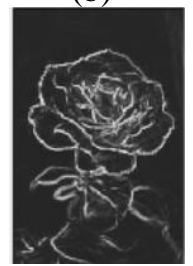

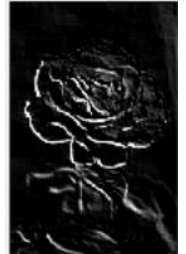

(c)

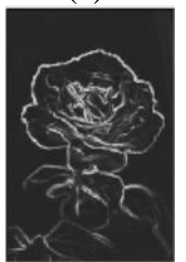

(f)
Figure. 12 Results of all methods for flower1 image:(a) original image, (b) ground truth, (c) Sobel method, (d) BSO method, (e) BFM method, and (f) Hybird method BSO-BFM

Table 6. Performance measures for flower1 image

\begin{tabular}{|c|l|l|c|}
\hline Methods & \multicolumn{1}{|c|}{$\boldsymbol{M S E}$} & \multicolumn{1}{|c|}{$\boldsymbol{R} \boldsymbol{M} \boldsymbol{E}$} & $\boldsymbol{P S N R}$ \\
\hline Sobel & 0.223485 & 0.472742 & 54.6383 \\
\hline BSO & 0.097518 & 0.31228 & 58.2399 \\
\hline $\boldsymbol{B F M}$ & 0.053216 & 0.230686 & 60.8703 \\
\hline BSO-BFM & 0.466766 & 0.216048 & 61.4398 \\
\hline
\end{tabular}

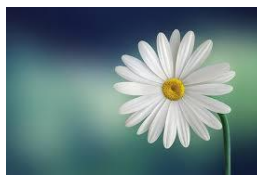

(a)

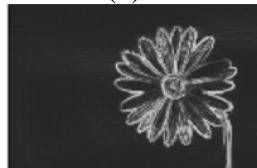

(d)

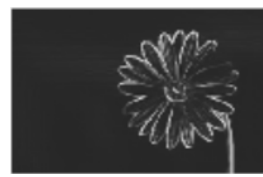

(b)

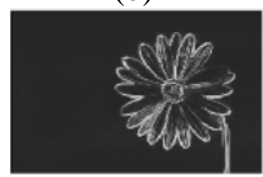

(e)

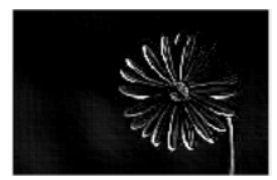

(c)

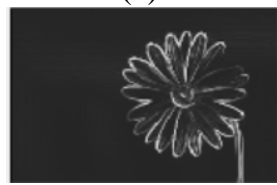

(f)
Figure. 13 Results of all methods for flower2 image: (a) original image, (b) ground truth, (c) Sobel method, (d) BSO method, (e) BFM method, and (f) Hybird method BSO-BFM

Table 7. Performance measures for flower2 image

\begin{tabular}{|c|c|c|c|}
\hline Methods & MSE & $\boldsymbol{R M S E}$ & $\boldsymbol{P S N R}$ \\
\hline Sobel & 0.150453 & 0.387883 & 56.3568 \\
\hline BSO & 0.082134 & 0.286592 & 58.9855 \\
\hline BFM & 0.063216 & 0.251428 & 60.1225 \\
\hline BSO-BFM & 0.046071 & 0.214642 & 61.4965 \\
\hline
\end{tabular}

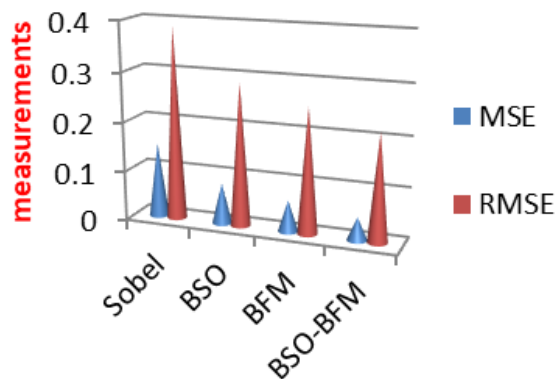

Figure. 14 MSE, RMSE measures of all methods

\section{Conclusion}

This research presents edge detection techniques and methods that depend on computational intelligence, and which are based in their work on biological content inspired by nature. Two swarm intelligence methods represented by the beetle optimization method and the butterfly optimization method for conducting the edge detection process were presented in the images. In addition, the main goal of the research was to develop a powerful edge detector based on the beetle and butterfly algorithms by improving the performance of the butterfly algorithm by selecting optimal values for its parameters that play an important role in finding the optimal solution. This was done by hybridizing it with the beetle algorithm that equips the butterfly algorithm with the best values for its parameters, and the hybridization process was done in first stage. The first stage of the butterfly method is the initialization stage.

The experimental results indicate that techniques based on swarm intelligence effectively increase the edges of image, compared to traditional techniques. Subjective results showed that the proposed BSOBFM method generated better-looking edges compared to traditional detectors such as, sobel. Objective simulations using MSE, RMSE and PSNR values also support the same results of BSO-BFM, the best results among other detectors used for research. Where when used sobel method the MSE values ranged between $0.135328-0.375440$, and the RMSE values between 0.36787- 0.612732, and PSNR values are 52.385397-56.8169, While in the proposed methods (beetle BSO, butterfly BFM, Hybrid method BSO-BFM) for determining and detecting the edges of the images, the MSE values ranged between 0.023375- 0.101380, and the RMSE values between $0.023375-0.101380$, and the PSNR values ranged between 58.071293 - 64.443273, as it was found that our research methods were the best in their results.

One of the future works that we recommend is to study swarm behaviors, and select algorithms with common characteristics, and cross-breed them to produce a new method for detecting edges. In addition to choosing pictures of a different color, as well as medical pictures to identify objects inside the picture

\section{Conflicts of Interest}

Declare conflicts of interest or state "The author declares no conflict of interest." Authors must identify and declare any personal circumstances or interest that may be perceived as inappropriately 
influencing the representation or interpretation of reported research results.

\section{Author Contributions}

For research articles with several authors, a short paragraph specifying their individual contributions must be provided. The following statements should be used as follows: "Conceptualization, Shahbaa; methodology, Shahbaa; software, Shahbaa; validation, Shahbaa; formal analysis, Shahbaa; investigation, Shahbaa; resources, Shahbaa; data curation, Shahbaa; writing - original draft preparation, Shahbaa; writing - review and editing, Shahbaa; visualization, Shahbaa; supervision, Shahbaa; project administration, Shahbaa; funding acquisition, Shahbaa", etc. Authorship must be limited to those who have contributed substantially to the work reported.

\section{Acknowledgments}

The Author would like to thank, the University of Mosul in Iraq, for Providing Moral Support During the Completion of this Research.

\section{References}

[1] R. Fatima, F. D. Mirajkar, H. Begum, and A. S. Math, "An Extensive Survey on Edge Detection Techniques", International Journal of Engineering and Techniques, Vol. 3, No. 6, pp. 63-69, 2017.

[2] A. Khunteta and D. Ghosh, "Edge Detection via Edge-Strength Estimation Using Fuzzy Reasoning and Optimal Threshold Selection Using Particle Swarm Optimization", Hindawi Publishing Corporation:Advances in Fuzzy Systems, Vol. 2014, pp. 1-17, 2014.

[3] B. I. Khaleel, "Image Clustering based on Artificial Intelligence Techniques", $A L$ Rafidain Journal of Computer Sciences and Mathematics, Vol. 11, No. 1, pp. 99-112, 2014.

[4] K. Raj, P. Gautam, K. Tiwari, and V. Goel, "A Review Paper: On Various Edge Detection Techniques", International Journal for Research in Applied Science \& Engineering Technology, Vol. 5, No. VIII, pp. 534-537, 2017.

[5] Y. Ren, "Image Edge Detection Based on Hybrid Algorithm", Boletín Técnico, Vol. 55, No. 17, pp. 997-1003, 2017.

[6] N. S. Dagar and P. K. Dahiya, "A Comparative Investigation into Edge Detection Techniques Based on Computational Intelligence", I.J. Image, Graphics and Signal Processing, Vol. 7, pp. 58-68, 2019.
[7] A. Eleyan and M. Anwar, "Multiresolution Edge Detection using Particle Swarm Optimization", International Journal of Engineering Science and Application, Vol. 1, No. 1, pp. 11-17, 2017.

[8] S. Kanugo and A. Mekala, "Particle Swarm Optimization based Edge Detection Algorithms for Computer Tomography Images", Indian Journal of Science and Technology, Vol. 9, No. 37, 2016.

[9] N. Dagar and P. Dahiya, "Edge Detection Technique using Binary Particle Swarm Optimization", Procedia Computer Science, Vol. 167, pp. 1421-1436, 2020.

[10] S. I. Khaleel and K. M. Saleh, "Detection of network anomaly based on hybrid intelligence techniques", AL-Rafidain Journal of Computer Sciences and Mathematics, Vol. 9, No. 2, pp. 81-98, 2012.

[11] R. Pushpalakshmi, P. Lavanya, and S. Bhuvaneshwari, "Survey of ACO and PSO based Secure Routing protocols for Wireless networks", International Journal of Advance Research in Science and Engineering, Vol. 6, No. 12, 2017.

[12] S. Khaleel, "Designing a Tool to Estimate Software Projects Based on The Swarm Intelligence", International Journal of Intelligent Engineering and Systems, Vol. 14, No. 4, pp. 524-538, 2021.

[13] B. I. Khaleel, "Using Artificial Intelligence Techniques for Image Compression", $A L$ Rafidain Journal of Computer Sciences and Mathematics, Vol. 11, No. 2, pp. 65-81, 2014.

[14] S. I. Khaleel and A. A. Thanoon, "Design a Tool for Generating Test Cases using Swarm Intelligence", AL-Rafidain Journal of Computer Sciences and Mathematics, Vol. 10, No. 1, pp. 421-444, 2013.

[15] C. Tao, H. Hua, S. Xiankun, and Y. Xiaoming, "Image Edge Detection based on ACO-PSO Algorithm", International Journal of Advanced Computer Science and Applications, Vol. 6, No. 7, 2015.

[16] S. I. Khaleel and R. Khaled, "Selection and Prioritization of Test Cases by using Bees Colony", AL-Rafidain Journal of Computer Sciences and Mathematics, Vol. 11, No. 1, pp. 179-201, 2014.

[17] R. Rai, R. Pradhan, and M. Ghos, "Ant based Swarm Computing Techniques for Edge Detection of Images- A Brief Survey", International Journal of Emerging Technology and Advanced Engineering, Vol. 3, No. 4, pp. 249-259, 2013. 
[18] N. Dagar and P. Dahiya, "Edge Detection Using Distinct Particle Swarm Optimization", International Journal of Innovative Technology and Exploring Engineering, Vol. 8, No. 9, pp. 1050-1057, 2019.

[19] D. Weikert, "2D-Contour Search using a Particle Swarm Optimization inspired Algorithm", Master Thesis, Otto-von-Guericke University, Magdeburg, 2019.

[20] L. Wang, Q. Wu, F. Lin, S. Li, and D. Chen, "A New Trajectory-Planning Beetle Swarm Optimization Algorithm for Trajectory Planning of Robot Manipulators", IEEE Access, Vol. 7, pp. 154331-154345, 2019.

[21] Y. Mu, B. Li, D. An, and Y. Wei, "ThreeDimensional Route Planning Based on the Beetle Swarm Optimization Algorithm", IEEE Access, Vol. 7, pp. 117804-117813, 2019.

[22] S. Khaleel, "Image Compression Using Swarm Intelligence", International Journal of Intelligent Engineering and Systems, Vol. 14, No. 1, pp. 257-269,2021.

[23] T. Chen, Y. Zhu, and J. Teng, "Beetle swarm optimisation for solving investment portfolio problems", The Journal of Engineering, Vol. 16, pp. 1600-1605, 2018.

[24] A. Eleyan and M. Anwar, "Multiresolution Edge Detection using Particle Swarm Optimization", International Journal of Engineering Science and Application, Vol. 1, No. 1, pp. 11-17, 2017.

[25] A. Mortazavi, "Comparative assessment of five metaheuristic methods on distinct problems", Dicle University Journal of Engineering, Vol. 10, No. 3, pp. 879-898, 2019.

[26] S. Arora and S. Singh, "Butterfly optimization algorithm: a novel approach for global optimization", Soft Computing, Vol. 23, pp. 715-734, 2019.

[27] S. Arora and S. Singh, "An Improved Butterfly Optimization Algorithm for Global Optimization", Advanced Science, Engineering and Medicine, Vol. 8, No. 9, pp. 1-7, 2016.

[28] G. Li, F. Shuang, P. Zhao, and C. Le, "An Improved Butterfly Optimization Algorithm for Engineering Design Problems Using the CrossEntropy Method", Symmetry, Vol. 11, pp. 1-20, 2019. 